\title{
Research on modeling and extraction of a dominant business process fragment
}

\author{
Wei $\operatorname{Tan}^{\dagger}$ \\ Guangdong Province Key Laboratory of Computer Network \\ South China University of Technology, Guangzhou 510641, China \\ e-mail: lxphmr@163.com; \\ Xuan Liu \\ Faculty of Economic and Management, Dongguan University of Technology \\ Dongguan, 523808, China \\ email:tanwphmr@163.com

\begin{abstract}
With the rise of the Internet plus, companies have embarked Internet plus road. To implement business process resource sharing is one of the main ways for enterprises to achieve Internet plus. Due to the participation of many enterprises, the business process resources on the Internet are massive. It is necessary to improve the competitiveness of its resources In order to make the customers choose their resources as much as possible. For this reason, this paper puts forward the advantage business process fragment model, and the extraction method is designed. Finally, the experimental results show that the method is effective and the validity of the method is verified.
\end{abstract}

Keywords: Internet plus; Dominant business process segment; Extraction

\section{Introduction}

Enterprises have many business processes, and each business process has a key process node or business process segment. The business process fragment is composed of process nodes which are closely connected with the flow relation. Key business process segment is the core of the business process, on which the quality of the finished product depends. In order to realize the resource sharing of the business process, and to enable customers to choose their process resources as much as possible, enterprises are bound to improve the competitiveness of their business process resources. The enterprise can select the dominant business process (single node is its special case) and can construct its advantage process resource fragment through their key business process resource fragment to improve the competitiveness of business process resources.

In business process modeling, document [1] proposed a business process conceptual model framework based on the goal. Bottom up reverse generation of 
flow fragments matching each sub target, and then the evaluation and analysis of the support of behavior and business process fragment to the target are given. Document[2]-[6] proposed respective manufacturing resource models for cloud manufacturing service. document [7] proposed a unified Unified model of multigranularity manufacturing resource.In terms of business process extraction, document [8] proposed a process recommendation technology based on process warping matrix in order to solve the deficiency of the current process recommendation technology in the field of fuzzy recommendation. document [9] proposed Process recommendation technique which used graph mining technique to extract the process patterns, document [10] combines the behavior profile into it On the basis of the process model mining method taken quasi indirect dependence. document[11] given method of the functional similarity and process similarity, document [12] designed a quantitative similarity evaluation algorithm, considering the static and dynamic characteristics of the combined process to improve the accuracy of service discovery.

Above studies have studied the modeling and extraction of business process, however, there is a lack of research on the advantages of process fragments.

In this paper, based on the actual business background and the status of its development, in-depth research on business processes have been done, including business process fragment modeling, business process fragment extraction.

\section{Advantage business process fragment modeling}

Definition 1. Business process activity node. It is a child node on the business process. Each node has a clear business function and its base information. The nodes are connected by business process relationship. Its formalization can be expressed as node=(base,func), where base express its base information, and base $=($ name, type,enterprise,...), that is resource's attribute information, func express its function, and func $=(\mathrm{I}, \mathrm{o})$, that is resource's input and output information.

Defintion 2. Business process relationship. It is the relationship between process nodes, generally including parallel $\mathrm{P}$, serial s, loop $\mathrm{C}$, branch structure $\mathrm{B}$ four relationships. The set of relations can be described as relation $=\{\mathrm{p}, \mathrm{s}, \mathrm{c}, \mathrm{b}\}$.

Fig.1 Business process relation
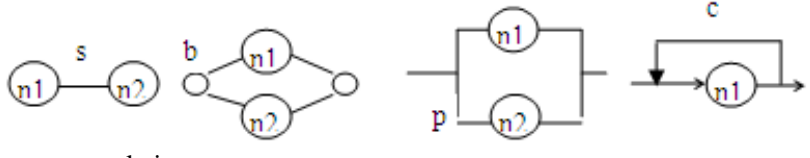

Definition 3. Business process string expression. It is an expression who describes the business process and its process relation in the form of string. Its 
description method uses symbolic function, for example, $\mathrm{S}$ as $\mathrm{S}($ ), and uses dot separate two nodes, uses parenthesis split two branches.

As shown in Figure 1, two nodes on the relationship of serial structures can be expressed s(n1.n2), the two nodes of the branching structure $b((n 1)(n 2))$, two node of the parallel structure $\mathrm{p}((\mathrm{n} 1)(\mathrm{n} 2))$, node of the loop structure $\mathrm{c}(\mathrm{n} 1)$.

Definition 4.Business process. It is a set of business process nodes with a certain business process relationship, which able to complete the business tasks. Its formal representation can be described as flow=(nodes,relation).

Defintion 5. Business process' quality of service. It refers to the quality which is evaluated for business process to complete the task. It is determined by many factors, generally including time $t$, cost $c$, reliability $r$, availability a, and can be formally expressed as BPqos $=(\mathrm{t}, \mathrm{c}, \mathrm{r}, \mathrm{a})$.

Difinition 6. Dominant business process segment. It is a segment of the process that plays a central role in the business process. This section of the process is outstanding role in the whole process of, and has strong competitiveness compared with similar resources, can be formally expressed as $\mathrm{DBPF}=($ flow, BPqos).

\section{Construct Of Advantage Process Fragment}

Definition 7 Business process set vector. $\mathbf{X}=\left(x_{1}, x_{2} \ldots, x_{i}, \ldots x_{n}\right), i=1,2, \ldots, n$,where $x_{i}$ expresses the business activity, $n$ expresses the number of activities. $\mathbf{W}^{x}=\left(w_{1}^{x}, w_{2}^{x}, \ldots, w_{j}^{x}, \ldots, w_{n}^{x}\right)$, $j=1,2, \ldots, n$, where $W^{x}$ expresses the weight vector of each business activity performance, $w_{j}^{x}$ expresses the weight of corresponding $x_{j}$. The higher the value, the higher business performance of the corresponding business activity. It satisfies $\sum_{j=1}^{n} w_{j}^{x}=1$.

Definition 8. Each business activity performance index vector. $\mathbf{Z}=\left(z_{1}, z_{2}, \ldots, z_{i}, \ldots, z_{m}\right), i=1,2, \ldots, m$,where $z_{i}$ expresses the $i$ business performance indicator, reflecting certain aspects of the performance of the activity, and $m$ expresses index number. $\mathbf{W}^{z}=\left(w_{1}^{z}, w_{2}^{z}, \ldots, w_{j}^{z}, \ldots, w_{m}^{z}\right), i=1,2, \ldots, m$, where $W^{z}$ expresses business activity index weight vector, and $w_{j}^{z}$ expresses weight corresponding to $z_{j}$, the higher the value, the more business performance in all the indexes the corresponding business activity performance index. It satisfies $\sum_{j=1}^{m} w_{j}^{z}=1$. 
Definition 9. The expert score vector. $\mathbf{F}_{i}^{x}=\left(f_{i 1}^{x}, f_{i 2}^{x}, \ldots f_{i j}^{x}, \ldots, f_{i m}^{x}\right)$, where $F_{i}^{x}$ expresses performance evaluation index vector of $x_{i}(i=1,2, \ldots, n)$, and $f_{i j}^{x}$ expresses the expert scoring average of $z_{j}$ of $x_{i}(j=1,2, \ldots, m)$, formulas are as followed:

$$
\begin{gathered}
w_{i}^{x}=\frac{\sum_{j=1}^{m} f_{i j}^{x} \cdot w_{j}^{z}}{\sum_{i=1}^{n} \sum_{j=1}^{m} f_{i j}^{x} \cdot w_{j}^{z}} \\
w_{j}^{z}=\frac{f_{i j}^{x}}{\sum_{j=1}^{m} f_{i j}^{x}}
\end{gathered}
$$

Where, score standard of business activities and resources can be assessed at the same level. Therefore, the following uniform standard can be adopted, namely, fuzzy evaluation method or superiority factor evaluation grade: very strong, strong, relatively strong, nearly, slightly inferior, relatively poor, poor, which can be allocated as rating numerical: 7, 6, 5, 4, 3, 2, 1. The higher the score, the more strong its advantage. Comprehensive performance of each activity in business process can be obtained by formula (1), (2).

The activity performance weight vector of the business process can be gotten based on the evaluation method of the core business activities. Values of the vector may be:

(1)Weight values around the height peak are larger

(2)One end of the height peak is larger

(3)Height peak is outlier

(4)No peaks but a whole is too large

A key problem of fragment extraction is how to intercept the fragment. Principles for intercept fragment are as following: one is maintaining the integrity of the process logic, two is the coupling, not separating but often binding activity, and three is higher whole performance. Here, calculation method of the overall performance value is done by calculating the average fragment performance.

The overall association extension extraction algorithm (OAEEA) is designed by taking different measures to extract according to the case of the vector value, and accounting for coupling process and the whole performance of the fragment, which extracts advantage fragment in business process. 
The algorithm process is as following:

(1) Firstly to calculate business process performance weight vector.

(2) Then to select the maximum weight value elements in the process called crest, if close values are too few,they are all selected. If there is no clear peak, there is a period of relatively large and smooth elements, which is extracted as an independent whole.

(3) In view of each maximal element, extract its upper and lower elements. For each extracted element, combined with the process logic of corresponding activity, A. for the upstream nodes, and the nodes are parallel node, all nodes in the parallel need to be extracted, for the selection structure, select the branch with large performance value. B. For the downstream node, also extract the parallel node, extract branch structure with large performance value, to maintain the integrity of the process logic. Expanded interception is done through combining with the often bundled activities, if value reaches the ends of slumping (In this study, each wave is seen as the calculating unit, and the average values of the wave is computed), it stops expansion, as far as possible to ensure the interception of the elements to achieve a higher performance value. C. For individual element, referring to the business logic, if it is not complete, then extended to complete, trying to extend the minimum range, if encountered in slumping, it stops. D. The isolated points are extracted as single granularity resource.

(4) Save the extracted edge fragment.

Pseudo code for OAEEA algorithm is presented as followed according to the above algorithm process.

Input:Businessx, $\{$ KunActiony $\mid \mathrm{y}=1, \ldots, \mathrm{n}\}$

Output: $\{$ Procz $\mid z=1, \ldots, m\}$

1. Input: Businessx

2. $W^{x}=$ Calculate $($ Business $x)$

\section{Bofeng=Select $\left(W^{x}\right)$}

4.for(int $\mathrm{i}=0 ; \mathrm{i}<\mathrm{k} ; \mathrm{i}++)$

5. $\{$ if(node)

6. $\{$ if(Up)

7. $\quad$ extend to up_boundary

8. get left_proc $\}$

9. \}

10. else

11. $\{$ extend to down_boundary 


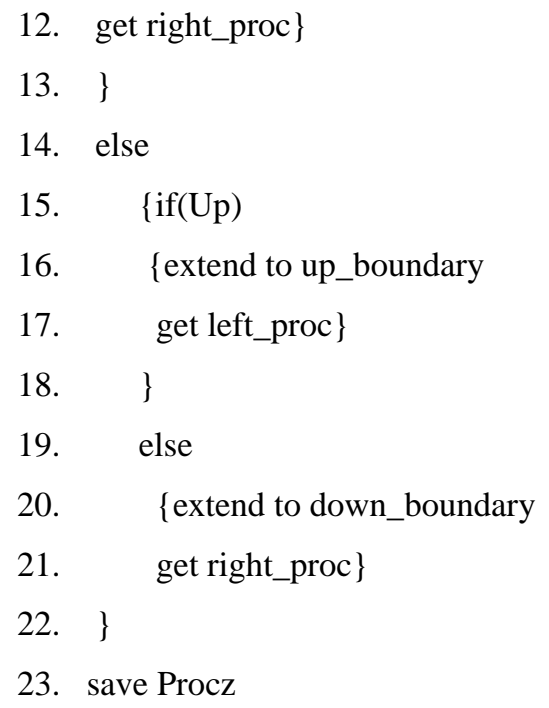

\section{4.. Experiment}

The following are examples of the solution process for comprehensive performance of business activities.

TABLE I. PROCESS SAMPLE

\begin{tabular}{llllllll}
\hline \multirow{2}{*}{ act } & expert & time & technology & $\begin{array}{l}\text { Production } \\
\text { quality }\end{array}$ & cost & important & $\begin{array}{l}\text { external } \\
\text { dependence }\end{array}$ \\
\hline \multirow{4}{*}{ x1 } & J1 & 5 & 5 & 6 & 5 & 3 & 3 \\
& J2 & 6 & 6 & 5 & 4 & 4 & 3 \\
& J3 & 4 & 5 & 5 & 6 & 3 & 2 \\
& J4 & 5 & 6 & 5 & 4 & 4 & 2 \\
& J5 & 4 & 5 & 6 & 4 & 3 & 3 \\
& J1 & 6 & 5 & 6 & 5 & 5 & 4 \\
x2 & J2 & 6 & 4 & 6 & 6 & 5 & 3 \\
& J3 & 7 & 5 & 5 & 6 & 5 & 4 \\
& J4 & 6 & 6 & 5 & 4 & 4 & 3 \\
& J5 & 5 & 5 & 6 & 4 & 6 & 3 \\
& J1 & 4 & 5 & 4 & 3 & 3 & 2 \\
x3 & J2 & 5 & 4 & 5 & 4 & 3 & 1 \\
& J3 & 4 & 5 & 5 & 3 & 3 & 2 \\
\hline
\end{tabular}

For example, if the business process is as shown in Figure 2, including three activities $\mathrm{x} 1, \mathrm{x} 2$ and $\mathrm{x} 3$, composed according to sequence. Their data are obtained according to the following procedure.

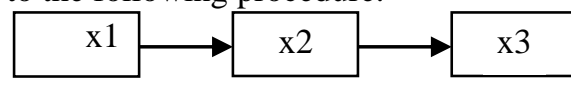

Fig.2 Business process example 
Firstly, business analysts establishes a performance index table, then the experts score, finally, average value of each index according to the expert scoring is calculated. The results are shown in Table 1.

$w_{j}^{z}$ Of the three activities can be calculated through formula (2), which are $\mathrm{x} 1(0.18,0.21,0.21,0.17,0.13,0.1)$,

$\mathrm{x} 2(0.2,0.17,0.18,0.17,0.17,0.11), \mathrm{x} 3(0.2,0.2,0.23,0.17,0.13,0.07)$ in turn. Finally, $w_{i}^{x}$ can be calculated through formula (1), results of which are 0.34 , $0.38,0.28$ respectively.

Advantage fragment of the example at Figure 3 is extracted based on the above algorithm. Extraction result is as followed. Its value of peak is 0.38 , caring on the expansion to it. Due to the business process is a simple sequential structure, the extension is simple. Extended to the left (belongs to type of the high end) for it, and done judgment in accordance with the sharp boundary, the value of the left element is in the boundary line. At the same time, combined with the binding information (If there is, it must be extracted), the left element is included into the extension set. While the right is under the boundary line, so stop to extend. Finally, an advantage fragment of $x 1 \times 2$ is gotten.

\section{Conclusion}

Under the background of Internet plus technology background and enterprise resource sharing requirement, an advantage business process segment model is proposed and its extraction algorithm is constructed. Through experiment, it is verified that extraction method is valid. The innovations of this paper are (1)a advantage business process fragment model is constructed,(2) an evaluation method of the advantages of business process and its extraction algorithm is constructed based on business process.

In the future, the further research on performance evaluation factors' selection and perfecting evaluation system will be done. Cloud service building study based on advantage business process fragment will be done for resource sharing based on Internal plus.

\section{Acknowledgements}

This work was financially supported by the National Natural Science Foundation of China under Grant No. 61070092, the combination of industry and research projects of the Ministry of Education in Guangdong Province under Grant No.2012B091100016, Guangdong Province Science and technology plan project under Grant No. 2015A010103022, 2014B090901002, 2014A010103039 and Science and technology project in Dongguan under Grant No. 2014509102211. 


\section{References}

[1] WANG Nan,SUN Shan-wu, OUYANG Dan-tong. Goal-based Conceptual Business Process Modeling[J].Computer Scince, 2014, 41(10):220-224.

[2] WEI Yun; LI Dongbo; TONG Yifei. Service-oriented Unified Manufacturing Resource Modeling Based on Meta-model [J]. China Mechanical Engineering, 2012, 23 (15):1818-1824.

[3] Linan ZHU, Yanwei ZHAO, Wanliang WANG. A Bilayer Resource Model for Cloud Manufacturing Services [J].Mathematic Problems in Engineering, 2013, 1-10.

[4] YAO Chang-feng, ZHANG Ding-hua, B UK un, et al. Networked manufacturing resources modeling and information integration based on physical manufacturing unit [J]. Computer Integrated Manufacturing Systems, 2008, 14(4):667-674.

[5] LIU, Ning; LI, Xiao-Ping; WANG, Qian. A resource \& capability virtualization method for cloud manufacturing systems [J], IEEE International Conference on Systems Man and Cybernetics Conference Proceedings, 2011, page: 1003-1008.

[6] SU Xijuan, XU Minghai, XU Yu, et al. A Virtual Resource Description and Discovery Model Based on Hybrid Granularity [J]. Telecommunications Science 2013, 2:43-50.

[7] TAN Wei,YAO Xi-Fan,LIU Xuan, et al.Unified model of multi-granularity manufacturing resource and its semantic service discovery [J].CHINA SCIENCEPAPER,2013,8(10):1044-1049.

[8] YE Yan-ming, YING Jian-wei, YIN CAO Bin.Process warping matrix based business process recommendation technique[J]. Computer Integrated Manufacturing Systems, 2013,19 (8):1868-1875.

[9] A Near Neighbour and Maximal Subgraph First Based Business Process Recommendation Technique[J].Chinese Journal of Computers, 2013,36(2):263-274.

[10] HUA Pei,FANG Xian-wen,LIU Xiang-wei.Method of Process Models Mining Based on Quasi Indirect Dependence[J].Computer Science, 2016,43(11):94-97.

[11] SUN Ping, JIANG Chang-Jun.Using Service Clustering to Facilitate Process-Oriented Semantic Web Service Discovery[J]. Chinese Journal of Computers, 2008,31(8):1340-1353.

[12] HUANG Zi-Cheng, HUAI Jin-Peng, LIU Xu-Dong,etc.Automatic Service Discovery Framework Based on Business Process Similarity[J]. Journal of Software,2012,23(3):489 -503. 